\title{
Elevated expression of RNA methyltransferase BCDIN3D predicts poor prognosis in breast cancer
}

\author{
Ling Yao ${ }^{1, *}$, Yayun $\mathrm{Chi}^{1, *}$, Xin $\mathrm{Hu}^{1, *}$, Shan $\mathrm{Li}^{1}$, Feng Qiao ${ }^{1}$, Jong $\mathbf{W u}^{1,2,3}$ and Zhi-Ming \\ Shao ${ }^{1,2,3}$ \\ ${ }^{1}$ Department of Breast Surgery, Fudan University, Shanghai Cancer Center and Cancer Institute, Shanghai, P.R. China \\ 2 Department of Oncology, Shanghai Medical College, Fudan University, Shanghai, P.R. China \\ ${ }^{3}$ Institutes of Biomedical Science, Fudan University, Shanghai, P.R. China \\ ${ }^{*}$ These authors have contributed equally to this work
}

Correspondence to: Zhi-Ming Shao, email: shaozmyl@126.com

Jong Wu, email: wujiong1122@vip.sina.com

Keywords: BCDIN3D, breast cancer, TNBC

Received: October 12, $2015 \quad$ Accepted: May 13, $2016 \quad$ Published: May 28, 2016

\begin{abstract}
Background: BCDIN3D is a member of the Bin3 methyl-transferase family that targets the 5' mono-phosphate of nucleic acids. Although BCDIN3D has been shown to increase tumorigenic phenotypes and invasiveness in MDA-MB-231 cells, its the clinical implications in breast cancer remain unclear. Methods: We screened for BCDIN3D using tissue microarrays constructed from 250 patients who were histologically confirmed to have invasive ductal breast carcinoma at the Fudan University Shanghai Cancer Center. Results: The survival analysis by Kaplan-Meier and Cox regression showed that BCDIN3D expression level served as a prognostic factor for disease-free survival $(P=\mathbf{0 . 0 4 2})$. The prognostic value of BCDIN3D was most significant in triplenegative breast cancer $($ TNBC) patients $(P=0.007)$. Conclusions: BCDIN3D might serve as an important prognostic factor for TNBC patients.
\end{abstract}

\section{BACKGROUND}

Breast cancer is the most common cancer in women, and account for approximately $23 \%$ of all cancer cases and approximately $14 \%$ of cancer deaths [1]. It is a heterogeneous disease embracing several different phenotypes [2], including luminal A, luminal $\mathrm{B}$, human epidermal growth factor receptor 2 (HER2)-enriched, and triple-negative breast cancer (TNBC) [3].Breast cancer results from the accumulation of genetic and epigenetic alterations [4, 5]. Epigenetic alterations, as defined by modifications of DNA, histones and coding/ noncoding RNAs [6,7], also contribute to various phases of neoplastic development including initiation, promotion, invasion, metastases.

RNA modification is a kind of epigenetic regulation of gene expression, analogous to DNA methylation and histone modification. [8]. Methylation is a ubiquitous modification that affects several residues/ sites in molecules [9]. Methyl-transfer reactions to RNA nucleotides are catalyzed by a variety of RNA-MTases that include more than 60 members with hundreds of homologs, and which have so far been divided into four super-families [10]. BCDIN3D, a member of the Bin3 methyltransferase family, share homology within their putative S-adenosyl Methionine (SAM) binding motif from S. pombe to human. SAM (S-adenosylmethionine) is well known as the methyl donor for methyl-transferases that modify DNA, RNA, histones [11].

BCDIN3D is a methyltransferase that targets the 5'mono-phosphate of nucleic acids. As depletion of BCDIN3D in the MDA-MB-231 cells abolishes anchorage-independent growth and decreases invasiveness in MDA-MB-231 cells, but not growth or migration [12], its prognostic value may be of interest. Herein, we investigated BCDIN3D expression and its association with tumor progression and clinical outcome in a cohort of 250 patients who had undergone surgery for breast cancer in eastern Chinese women population. 


\section{RESULTS}

\section{Patient characteristics}

Clinical-pathological characteristics of the study cohort are summarized in Table 1. Patients' median age at diagnosis was 52.02 years. After a mean follow-up time of 83.36 months, 55 of the 250 patients showed recurrence of disease. In univariate analysis, histological grade (HR, 1.761; 95\% CI, 1.041-2.980; $P=0.035)$, tumor size (HR, 1.715; 95\% CI, 1.086-2.708; $P=0.021$ ), metastatic nodes (HR, 2.231;95\% CI, 1.304-3.819; $P=0.003$ ), were significantly associated with higher risk of recurrence and reached statistical significance as expected, however, only the association with tumor size and metastatic nodes remained statistically significant in multivariate analysis (Table 3).

\section{Expression patterns of BCDIN3D in breast cancer patients}

In this cohort of 250 patients, TMAs were immunostained for BCDIN3D (representative images, Figure 1). The specificity of the antibody against BCDIN3D was confirmed by Western blot of human cell lysis. Positive staining of BCDIN3D was detected in $49.6 \%$ of tumors according to the scoring criterion described above ( $n=124 ; 49.6 \%$ positive, $50.4 \%$ negative; Figure 1a-1d). We further analyzed relationships between clinicopathologic features and the expression level of BCDIN3D. The percentages of positive staining of protein were consistent across all subsets of patients (Table 2).

\section{BCDIN3D was identified as a significant prognostic factor in breast cancer patients, especially in triple-negative breast cancers (TNBC)}

Both univariate and adjusted multivariate survival analyses showed significant differences in DFS between the BCDIN3D positive and negative groups. BCDIN3D positive group having a significantly higher incidence of disease events in both univariate analysis $(\mathrm{HR}=1.754$; 95\% CI: 1.012-3.039; $P=0.045)$ and multivariate analysis. $(\mathrm{HR}=1.904 ; 95 \% \mathrm{CI}: 1.081-3.354 ; P=0.026)$ (Table 3$)$. The BCDIN3D positive group also showed worse DFS in the Kaplan-Meier survival analysis $(P=0.042$; Figure 2a). Thus, these results indicate that BCDIN3D expression is direct associated with breast cancer recurrence. Then we analyzed the relationship between BCDIN3D expression and survival according to the different breast cancer subtypes. We found the prognostic value of BCDIN3D for
DFS was most significant among patients with TNBC ( $P$ $=0.007$; Figure $2 \mathrm{~b}$ ). In the TNBC subset, patients with positive BCDIN3D staining showed a higher likelihood of occurrence of disease events (HR $=3.584 ; 95 \% \mathrm{CI}$ : 1.319 9.737; $P=0.012)$ in univariate analysis and remain the same trend in multivariate analysis. $(\mathrm{HR}=3.719 ; 95 \% \mathrm{CI}$ : 1.345-10.283; $P=0.011)$ (Table 4).

\section{DISCUSSION}

Breast Cancer, is now known to involve epigenetic abnormalities along with genetic alterations [13]. Epigenetic modifications are early events in breast carcinogenesis and could be useful for early detection, prognosis, and targeted therapy of breast cancer [14]. Post-transcriptional gene regulation by noncoding RNA commonly referred as microRNAs is a kind of epigenetic regulation $[15,16]$. It has become one of the most dynamic and fast-growing fields in science. Despite the rapid advance of RNA research, the enzymes that posttranscriptionally modify RNA have been less investigated [17].

A post-transcriptional modification enzyme BCDIN3D O-methylates the $5^{\prime}$ terminal mono-phosphate group of the precursor of some miRNA, which increased tumorigenic phenotypes and cells invasiveness [12]. To our knowledge, no one else has explored its clinical significance. Our study is the first to evaluate the prognostic value of BCDIN3D in breast cancer patients. Our finding that patients with BCDIN3D positive tumors had worse DFS than the BCDIN3D negative group supports previous biological function study results from other researchers. Interestingly, this association was found to be most significant among patients with TNBC. TNBC breast tumors lack ER, PR, and HER-2 expression and occupy $15-20 \%$ of all breast cancers. It is generally more aggressive, has higher rates of relapse and decreased overall survival [18]. As few biomarkers are widely considered to be predictive for TNBC prognosis, and thus predictive factors for TNBC are urgently need. Our findings suggest that BCDIN3D might serve as an important prognostic factor for TNBC patients.

Our study had several limitations, including the small data set, few cases of stage I patients, lack of validation in an independent series of cases, and the composition of the study cohort which did not exactly represent that of the breast cancer population. However, our study was based on the use of TMAs, which can guarantee the consistency and coherence of these factors.

\section{CONCLUSIONS}

In conclusion, our results associate, for the first time, higher BCDIN3D levels with worse DFS, especially in triple-negative breast cancer population, which suggests 
Table 1: Clinical-pathological characteristics of the study cohort

\begin{tabular}{|c|c|c|}
\hline Clinical-pathological characteristics & No. & Percentage (\%) \\
\hline \multicolumn{3}{|c|}{ Age (vears) (mean 52.02. SD 9.642. median 51, range 29-85) } \\
\hline$<50$ & 120 & 48.0 \\
\hline$>50$ & 130 & 52.0 \\
\hline \multicolumn{3}{|l|}{ Menopausal status } \\
\hline Pre & 108 & 43.2 \\
\hline Post & 142 & 56.8 \\
\hline \multicolumn{3}{|l|}{ TNM Stage } \\
\hline $\mathrm{I}$ & 74 & 29.6 \\
\hline U & 132 & 52.8 \\
\hline$山$ & 42 & 16.8 \\
\hline Unknown & 2 & 0.8 \\
\hline \multicolumn{3}{|l|}{ Histological grade } \\
\hline $\mathrm{I}$ & 5 & 2.0 \\
\hline $\mathrm{U}$ & 184 & 73.6 \\
\hline III & 61 & 24.4 \\
\hline \multicolumn{3}{|l|}{ Tumor size } \\
\hline $\mathrm{T} 1(<2 \mathrm{~cm})$ & 115 & 46.0 \\
\hline $\mathrm{T} 2(>2-5 \mathrm{~cm})$ & 120 & 48.0 \\
\hline $\mathrm{T} 3(>5 \mathrm{~cm})$ & 13 & 5.2 \\
\hline Unknown & 2 & 0.8 \\
\hline \multicolumn{3}{|l|}{ Node status } \\
\hline Negative & 151 & 60.4 \\
\hline Positive & 97 & 38.8 \\
\hline Unknown & 2 & 0.8 \\
\hline \multicolumn{3}{|l|}{ ER status } \\
\hline Negative & 143 & 57.2 \\
\hline Positive & 106 & 42.4 \\
\hline Unknown & 1 & 0.4 \\
\hline \multicolumn{3}{|l|}{ PR status } \\
\hline Negative & 184 & 73.6 \\
\hline Positive & 63 & 25.2 \\
\hline Unknown & 3 & 1.2 \\
\hline \multicolumn{3}{|l|}{ HER-2 status } \\
\hline Negative & 148 & 59.2 \\
\hline Positive & 101 & 40.4 \\
\hline Unknown & 1 & 0.4 \\
\hline \multicolumn{3}{|l|}{ Molecular subtype ${ }^{a}$} \\
\hline Luminal like & 106 & 42.4 \\
\hline HER-2 Positive & 42 & 16.8 \\
\hline Triple Negative & 100 & 40.0 \\
\hline Unknown & 2 & 0.8 \\
\hline
\end{tabular}

Abbreviations: ER, estrogen receptor; PR, progesterone receptor; HER-2, human epidermal growth factor receptor 2; SD, standard deviation;

a. Definition of breast cancer molecular subtypes: luminal like (ER and/or PR positive, any HER-2 status), HER-2 Positive (ER and PR negative, HER-2 positive) and triple negative (ER negative, PR negative and HER-2 negative) 
its potential use as a predictive biomarker. Furthermore, as more future basic and clinical studies uncover the underlying mechanism in this pattern, BCDIN3D could emerge as a desperately needed therapeutic targetin triplenegative breast cancer.

\section{MATERIALS AND METHODS}

\section{Patients and specimens}

250 primary breast cancer samples of stage I to III invasive ductal carcinoma cases were collected randomly at the Department of Breast Surgery in Fudan University Shanghai Cancer Center (FDUSCC, Shanghai, P.R. China) between August 2001 and March 2006. Their clinicalpathological characteristics and the systemic therapies are presented in Table 1. In this retrospective cohort study, they have been followed regularly, and 227 cases obtained the clinical outcome, with the last update in September 2013. Their median follow-up time was 96 months.

\section{Ethics statement}

This study was approved by the Ethics Committee of FDUSCC, and each participant signed an informed consent document.

\section{Tissue microarrays (TMAs)}

TMAs were constructed from formalin-fixed, paraffin-embedded samples of carcinomas obtained from the 250 breast cancer patients. A hematoxylin- and eosinstained section of each tumor block was used to mark representative tumor regions. Tissue cylinders with a diameter of $2 \mathrm{~mm}$ were punched from the above regions and transferred to recipient array blocks using a Tissue Micro Arrayer (Beecher Instruments, Sliver Springs, MD, USA). TMAs were composed of duplicate cores from different areas of the same tumor to compare staining patterns.

\section{IHC experimental procedures}

The tissue micro arrays were subjected to immunohistochemical staining for BCDIN3D, using a 2-step protocol (GTVisionTMIII). The primary antibodies used were monoclonal BCDIN3D antibody (Santa Cruz Biotechnology, CA, USA sc-390348). The specificity of the BCDIN3D antibody was validated by western blot (Figure S1). The TMAs were deparaffinized with xylene, and rehydrated with an ethanol gradient. The sections were then rinsed with phosphate buffer solution (PBS) for immunohistochemical staining. For antigen retrieval the sections were immersed in 0.01

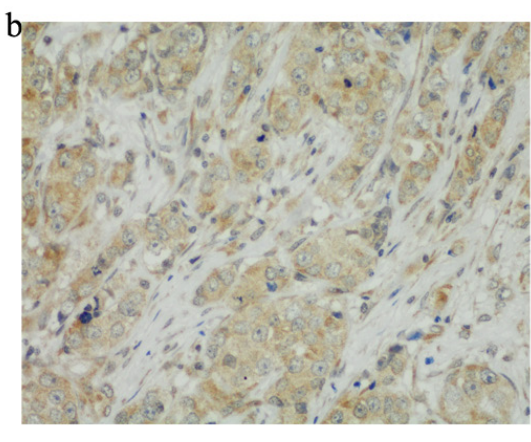

$40 \times$

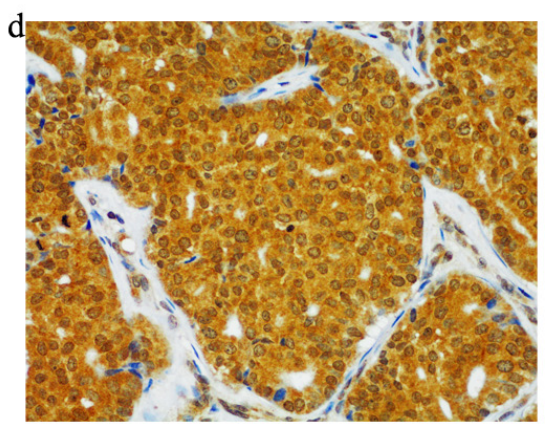

$40 \times$

Figure 1: Representative image of immunohistochemical BCDIN3D staining were shown in both small pictures $(\times 100$ magnification) and large (×400 magnification). a.-b. Negative for BCDIN3D. c.-d. Positive for BCDIN3D. 
Table 2: Correlations between patients' characteristics and expression status of BCDIN3D

\begin{tabular}{|c|c|c|c|c|}
\hline \multirow[b]{2}{*}{ Clinical-pathological characteristics } & \multirow[b]{2}{*}{ Cases } & \multicolumn{3}{|c|}{ BCDIN3D } \\
\hline & & - & + & $P^{a}$ \\
\hline & 250 & 126 & 124 & \\
\hline \multicolumn{2}{|l|}{ Percentage $(\%)$} & $50.40 \%$ & $49.60 \%$ & \\
\hline \multicolumn{5}{|l|}{ Age(years) } \\
\hline$\leq 50$ & 120 & 62 & 58 & \multirow{2}{*}{0.521} \\
\hline$>50$ & 130 & 64 & 66 & \\
\hline \multicolumn{5}{|l|}{ Menopausal status } \\
\hline Pre & 108 & 54 & 54 & \multirow{2}{*}{1.000} \\
\hline Post & 142 & 72 & 70 & \\
\hline \multicolumn{5}{|l|}{ TNM Stage } \\
\hline $\mathrm{I}$ & 74 & 32 & 42 & \multirow{3}{*}{0.250} \\
\hline II & 132 & 73 & 59 & \\
\hline III & 42 & 21 & 21 & \\
\hline \multicolumn{5}{|l|}{ Histological grade } \\
\hline I & 5 & 1 & 4 & \multirow{3}{*}{0.334} \\
\hline II & 184 & 92 & 92 & \\
\hline III & 61 & 33 & 28 & \\
\hline \multicolumn{5}{|l|}{ Tumor size } \\
\hline $\mathrm{T} 1(\leq 2 \mathrm{~cm})$ & 115 & 52 & 63 & \multirow{3}{*}{0.318} \\
\hline $\mathrm{T} 2(>2-5 \mathrm{~cm})$ & 120 & 66 & 54 & \\
\hline $\mathrm{T} 3(>5 \mathrm{~cm})$ & 13 & 8 & 5 & \\
\hline \multicolumn{5}{|l|}{ Node status } \\
\hline Negative & 151 & 75 & 76 & \multirow{2}{*}{0.697} \\
\hline Positive & 97 & 51 & 46 & \\
\hline \multicolumn{5}{|l|}{ ER status } \\
\hline Negative & 143 & 69 & 74 & \multirow{2}{*}{0.522} \\
\hline Positive & 106 & 56 & 50 & \\
\hline \multicolumn{5}{|l|}{ PR status } \\
\hline Negative & 184 & 88 & 96 & \multirow{2}{*}{0.243} \\
\hline Positive & 63 & 36 & 27 & \\
\hline \multicolumn{5}{|l|}{ HER-2 status } \\
\hline Negative & 148 & 78 & 70 & \multirow{2}{*}{0.368} \\
\hline Positive & 101 & 47 & 54 & \\
\hline
\end{tabular}

Abbreviations: ER, estrogen receptor; PR, progesterone receptor; HER-2, human epidermal growth factor receptor 2; a. Pearson $\chi 2$ test 
Table 3: Univariate and multivariate analysis of factors related to disease-free survival (DFS) in breast cancer patients

\begin{tabular}{|l|l|l|l|l|}
\hline \multirow{2}{*}{ Variables } & \multicolumn{2}{l}{ DFS } \\
\cline { 2 - 5 } & \multicolumn{2}{l}{ Univariate Analysis } & \multicolumn{2}{l|}{ Multivariate Analysis } \\
\cline { 2 - 5 } & HR(95\% CIs) & $\boldsymbol{P}$ & \multicolumn{1}{l|}{ HR(95\% CIs) } & $\boldsymbol{P}$ \\
\hline Age & $0.815(0.552-1.534)$ & 0.939 & $0.971(0.554-1.700)$ & 0.917 \\
\hline Histological grade & $1.761(1.041-2.980)$ & $\mathbf{0 . 0 3 5}$ & $1.749(0.965-3.170)$ & 0.065 \\
\hline Tumor Size & $1.715(1.086-2.708)$ & $\mathbf{0 . 0 2 1}$ & $1.648(1.042-2.606)$ & $\mathbf{0 . 0 3 3}$ \\
\hline Metastatic nodes & $2.231(1.304-3.819)$ & $\mathbf{0 . 0 0 3}$ & $2.158(1.236-3.769)$ & $\mathbf{0 . 0 0 7}$ \\
\hline ER & $0.786(0.456-1.356)$ & 0.388 & $1.491(0.713-3.117)$ & 0.288 \\
\hline PR & $0.408(0.185-0.903)$ & $\mathbf{0 . 0 2 7}$ & $0.372(0.144-0.960)$ & $\mathbf{0 . 0 4 1}$ \\
\hline HER-2 & $0.936(0.548-1.600)$ & 0.809 & $0.754(0.412-1.378)$ & 0.358 \\
\hline BCDIN3D & $1.754(1.012-3.039)$ & $\mathbf{0 . 0 4 5}$ & $1.904(1.081-3.354)$ & $\mathbf{0 . 0 2 6}$ \\
\hline
\end{tabular}

Abbreviations: HR, hazard ratio; CIs, confidence intervals; ER, estrogen receptor; PR, progesterone receptor; HER-2, human epidermal growth factor receptor 2 ;

Table 4: Univariate and multivariate analysis of factors related to disease-free survival (DFS) in TNBC patients

\begin{tabular}{|c|c|c|c|c|}
\hline \multirow{3}{*}{ Variables } & \multicolumn{4}{|l|}{ DFS } \\
\hline & \multicolumn{2}{|l|}{ Univariate Analysis } & \multicolumn{2}{|l|}{ Multivariate Analysis } \\
\hline & HR(95\% CIs) & $P$ & HR(95\% CIs) & $P$ \\
\hline Age & $0.967(0.417-2.238)$ & 0.937 & $1.192(0.503-2.824)$ & 0.690 \\
\hline Histological grade & $1.273(0.553-2.927)$ & 0.570 & $0.953(0.392-2.315)$ & 0.915 \\
\hline Tumor Size & $2.837(1.381-5.825)$ & 0.005 & $1.950(1.023-3.719)$ & 0.042 \\
\hline Metastatic nodes & $3.195(1.364-7.486)$ & 0.007 & $3.157(1.265-7.880)$ & 0.014 \\
\hline BCDIN3D & $3.584(1.319-9.737)$ & 0.012 & $3.719(1.345-10.283)$ & 0.011 \\
\hline
\end{tabular}

Abbreviations: HR, hazard ratio; CIs, confidence intervals; ER, estrogen receptor; PR, progesterone receptor; HER-2, human epidermal growth factor receptor 2 ;
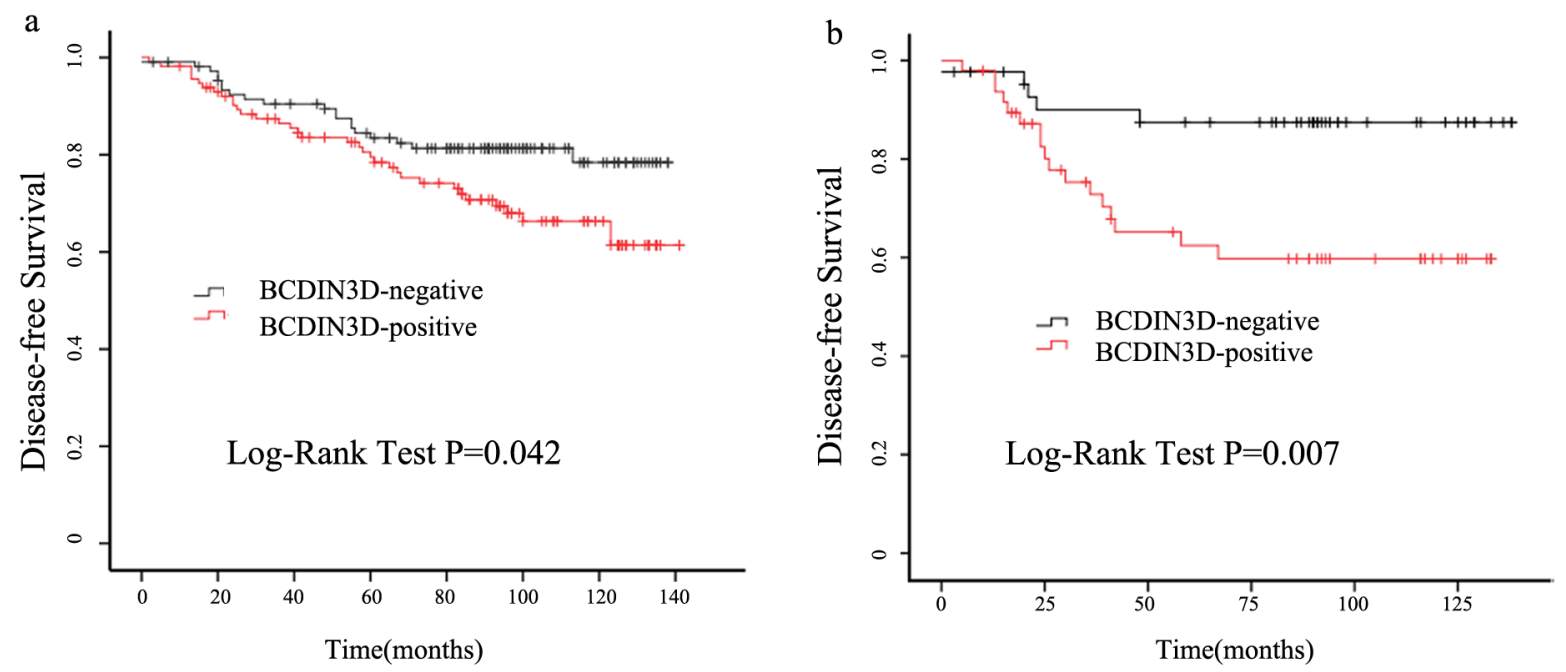

Figure 2: Elevated expression of BCDIN3D predicts worse clinical outcome in breast cancer patients, especially in TNBC. a. Cumulative disease-free survival curves of patients with positive or negative expression of BCDIN3D in breast cancer patients. b. Cumulative disease-free survival curves of patients with positive or negative expression of BCDIN3D in TNBC patients. 
$\mathrm{M}$ Tris sodium citrate $\mathrm{pH} 6.0$ and boiled at $121{ }^{\circ} \mathrm{C}$ for $5 \mathrm{~min}$ followed by $2 \mathrm{~min}$ simmering. All slides were incubated with nonspecific staining blocking agent for 20 minutes to quench endogenous peroxidase activity and then with anti-BCDIN3D $(1: 200)$ at $4{ }^{\circ} \mathrm{C}$ overnight. Primary antibodies were detected by HRP-conjugated secondary antibodies followed by colorimetric detection with 3, 3-diaminobenzidine (DAB). The TMAs were then counterstained with Gill hematoxylin and dehydrated in an ascending ethanol series before clearing with xylene and mounting under a coverslip.

\section{Evaluation of immunohistochemical variables}

TMAs were stained and scored semi-quantitatively. The score used in all subsequent analysis was the average across the available cores. Staining was graded for intensity of staining ( 0 , no staining; $1+$, faint/equivocal; $2+$, moderate; $3+$, strong) and percentage of cells stained $(0$, no staining; $1+,<10 \%$ of cells stained; $2+, 10 \%-50 \%$ of cells; and $3+,>50 \%$ of cells stained). For this study, SI $\geq 3$ was defined as positive staining, and $\mathrm{SI} \leq 2$ was defined as negative staining. Scoring was reviewed in parallel by two experienced breast disease pathologists who were blinded to all clinical data.

\section{Statistical analyses}

Disease-free survival(DFS) was defined as the time between the date of the primary surgery to the date of relapse/breast cancer specific death or September 2013. The first recurrence of disease at a local, regional, or distant site; diagnosis of contralateral breast cancer; and the breast cancer specific death were considered DFS events. Patients with study end date and loss of followup were considered to be censored. Correlations between clinical-pathological parameters and BCDIN3D were tested using the Chi-squared test. Survival outcomes were estimated using the Kaplan-Meier method and were compared between the groups using log-rank statistics. Univariate and multivariate analyses were carried out using the Cox risk proportion model. Statistics was analyzed using SPSS (version 13.0; SPSS Company). All $\mathrm{P}$ values are two-sided; $\mathrm{P}$ value less than 0.05 was considered significant. All analyses were based on the observed data with the assumption that missing data were completely at random.

\section{ACKNOWLEDGMENTS}

This research was supported by grants from the National Natural Science Foundation of China ( 81202082 ). These funding agencies had no role in study design, data collection and analysis, decision to publish, or preparation of the manuscript.

\section{CONFLICTS OF INTERESTS}

The authors declare that they have no competing interests.

\section{Authors' contributions}

ZS, JW conceived and designed the study. LY, YC and $\mathrm{XH}$ performed the experiments and analyzed the data. LS and FQ contributed reagents, materials, and analysis tools. All authors read and approved the final manuscript. All authors read and approved the final manuscript.

\section{REFERENCES}

1. Jemal A, Bray F, Center MM, Ferlay J, Ward E and Forman D. Global cancer statistics. CA Cancer J Clin. 2011; 61:6990.

2. Perou CM, Jeffrey SS, van de Rijn M, Rees CA, Eisen MB, Ross DT, Pergamenschikov A, Williams CF, Zhu SX, Lee JC, Lashkari D, Shalon D, Brown PO and Botstein D. Distinctive gene expression patterns in human mammary epithelial cells and breast cancers. Proc Natl Acad Sci U S A. 1999; 96:9212-9217.

3. Goldhirsch A, Winer EP, Coates AS, Gelber RD, PiccartGebhart M, Thurlimann B and Senn HJ. Personalizing the treatment of women with early breast cancer: highlights of the St Gallen International Expert Consensus on the Primary Therapy of Early Breast Cancer 2013. Ann Oncol. 2013; 24:2206-2223.

4. Kanwal R and Gupta S. Epigenetic modifications in cancer. Clin Genet. 2012; 81:303-311.

5. Virani S, Colacino JA, Kim JH and Rozek LS. Cancer epigenetics: a brief review. ILAR J. 2012; 53:359-369.

6. Xhemalce B. From histones to RNA: role of methylation in cancer. Brief Funct Genomics. 2013; 12:244-253.

7. Huang Y, Nayak S, Jankowitz R, Davidson NE and Oesterreich S. Epigenetics in breast cancer: what's new? Breast Cancer Res. 2011; 13:225.

8. Liu N and Pan T. RNA epigenetics. Transl Res. 2014.

9. Sibbritt T, Patel HR and Preiss T. Mapping and significance of the mRNA methylome. Wiley Interdiscip Rev RNA. 2013; 4:397-422.

10. Frankel AD and Smith HO. Restriction and modification enzymes detect no allosteric changes in DNA with bound lac repressor or RNA polymerase. J Mol Biol. 1981; 146:611-619.

11. Loenen WA. S-adenosylmethionine: jack of all trades and master of everything? Biochem Soc Trans. 2006; 34:330333.

12. Xhemalce B, Robson $\mathrm{SC}$ and Kouzarides T. Human RNA methyltransferase BCDIN3D regulates microRNA processing. Cell. 2012; 151:278-288. 
13. Berger SL, Kouzarides T, Shiekhattar R and Shilatifard A. An operational definition of epigenetics. Genes Dev. 2009; 23:781-783.

14. Dworkin AM, Huang TH and Toland AE. Epigenetic alterations in the breast: Implications for breast cancer detection, prognosis and treatment. Semin Cancer Biol. 2009; 19:165-171.

15. Ducasse $M$ and Brown MA. Epigenetic aberrations and cancer. Mol Cancer. 2006; 5:60.

16. Kanwal R and Gupta S. Epigenetics and cancer. J Appl Physiol (1985). 2010; 109:598-605.

17. He C. Grand challenge commentary: RNA epigenetics? Nat Chem Biol. 2010; 6:863-865.
18. Bastien RR, Rodriguez-Lescure A, Ebbert MT, Prat A, Munarriz B, Rowe L, Miller P, Ruiz-Borrego M, Anderson D, Lyons B, Alvarez I, Dowell T, Wall D, Segui MA, Barley L, Boucher KM, et al. PAM50 breast cancer subtyping by RT-qPCR and concordance with standard clinical molecular markers. BMC Med Genomics. 2012; 5:44. 